\title{
Numerical Solution of Klein/Sine-Gordon Equations by Spectral Method Coupled with Chebyshev Wavelets
}

\author{
Javid Iqbal, Rustam Abass \\ Department of Mathematical Sciences, BGSB University, Rajouri, India \\ Email: javid2iqbal@gmail.com, rustamabass13@gmail.com
}

How to cite this paper: Iqbal, J. and Abass, R. (2016) Numerical Solution of Klein/SineGordon Equations by Spectral Method Coupled with Chebyshev Wavelets. Applied Mathematics, 7, 2097-2109.

http://dx.doi.org/10.4236/am.2016.717167

Received: September 5, 2016

Accepted: November 12, 2016

Published: November 15, 2016

Copyright (c) 2016 by authors and Scientific Research Publishing Inc. This work is licensed under the Creative Commons Attribution International License (CC BY 4.0).

http://creativecommons.org/licenses/by/4.0/ cc) (i) Open Access

\begin{abstract}
The basic aim of this paper is to introduce and describe an efficient numerical scheme based on spectral approach coupled with Chebyshev wavelets for the approximate solutions of Klein-Gordon and Sine-Gordon equations. The main characteristic is that, it converts the given problem into a system of algebraic equations that can be solved easily with any of the usual methods. To show the accuracy and the efficiency of the method, several benchmark problems are implemented and the comparisons are given with other methods existing in the recent literature. The results of numerical tests confirm that the proposed method is superior to other existing ones and is highly accurate.
\end{abstract}

\section{Keywords}

Chebyshev Wavelets, Spectral Method, Operational Matrix of Derivative, Klein and Sine-Gordon Equations, Numerical Simulation, MATLAB

\section{Introduction}

Many physical phenomena encountered in science and engineering are governed by ordinary as well as partial differential equations. Some disciplines that use partial differential equations to describe the phenomena of interest are fluid mechanics, solid mechanics, quantum mechanic, propagation of acoustic and electromagnetic waves and problems in heat and mass transfer. Many linear and nonlinear phenomena appear in several areas of scientific fields like physics, chemistry and biology can be modeled by different type of partial differential equation such as evolution equation, reaction diffusion equation, Schrodinger type wave equations, Vander Poll's equation, Telegraph equation, Lyapunov equation etc. A broad class of analytical methods and numerical 
methods available in the literature are used to handle these problems. In this present work we are dealing with two partial differential equation named as Klein-Gordon and Sine-Gordon equations. The Klein-Gordon equation is as follows:

$$
u_{t t}-\alpha u_{x x}-\beta u_{y y}+g(u)=f(x, y),(x, y) \in \Omega, t \geq 0
$$

where $u=u(x, t)$ represents the wave displacement at position $x$ and time $t, \alpha$ and $\beta$ are known constant, $g(u)$ is the given nonlinear force and $f$ is the known function. If we assign the nonlinear force $g(u)=\sin u$ in (1) then it is known as SineGordon equation. The Klein-Gordon equation plays an important role in mathematical physics [1] [2] [3] and attracted more attention from scientists and engineering in different matter like investigation of the interaction of solutions in a collisionless plasma, the recurrence of initial states and examination of the nonlinear wave equations, studying the solutions and condensed matter physics and relativistic physics as a model of dispersive phenomena. On the other hand, Sine-Gordon equations appeared in many physical problems like applications in relativistic field theory, Josephson junctions or mechanical transmission lines [4] [5] [6] [7]. Numerical solution of partial differential equations is far more demanding than the ordinary ones. Several analytical or numerical methods such as decomposition method [8], variational iteration method [9], He's variational iteration method [10], collocation and radial basis functions [11], auxiliary equation method [12], spectral method [13] [14] [15], wavelet method [16] [17] [18] and the references therein have been proposed for the numerical solution of these types of equations. Among all these method mentioned above, spectral and wavelet method has got more attention of researcher from the last two decades.

Wavelet analysis had made a lot of successes in different fields of science and engineering due to its beautiful properties such as orthogonality, multi-resolution analysis and computational efficiency. Wavelet permits the accurate representation of a variety of functions and operators. Wavelet analysis and wavelet transform are recently developed mathematical tool for solving the linear and non-linear ordinary differential equations, partial differential equations and integral equation. Wavelets also applied in numerous disciplines such as image compression, data compression and deionising data. Most commonly wavelets are Haar, Legendre, Chebyshev are used to find the numerical solution of partial differential equations. In addition wavelet approach can make a connection with some fast and reliable numerical methods. The spectral method has the advantage of exponential convergence property when orthogonal basis functions are involved. As a result, it plays a vital role in solving partial differential equation. It is important to choose the basis function for possible coupling with spectral method. The wavelet basis can combine the advantages of both infinitely differentiable and small compact support which is far better than the spectral and finite element basis.

In recent year, spectral method [19] [20] using Legendre polynomials and Legendre wavelets as basic functions are considered to solve the Klein-Gordon and Sine-Gordon equations. By inspiring the work done in [19] [20], we use the Chebyshev wavelet as basis function coupled with spectral method for solving nonlinear Klein-Gordon and Sine-Gordon equations. Therefore, spectral collocation methods based on Chebyshev 
wavelet basis can obtain good spatial and spectral resolution while still keeping high efficiency.

The rest of the paper is as follows: In Section 2, Chebyshev wavelet and its properties are discussed. Operational matrix of derivative required for our subsequent development is presented in Section 3. Section 4 is devoted to present the Chebyshev wavelets spectral collocation method for solving Klein-Gordon and Sine-Gordon equations then approximate the unknown function. Section 5 deals with the illustrative examples and their solutions by the proposed approach compared with exact as well as with existing literature. Finally, concluding remarks are made in Section 6.

\section{Wavelets and Chebyshev Wavelets}

In the past decades, wavelets [21] [22] [23] shows their interest in different fields of science and technology due to its beautiful properties. Wavelets constitute the family of functions constructed from the dilation and translation of a single function known as the Mother wavelet. When the dilation parameter $a$ and translation parameter $b$ vary continuously we have the following family of continuous wavelets [23]

$$
\psi_{a, b(t)}=|a|^{-\frac{1}{2}} \psi\left(\frac{t-b}{a}\right) ; \quad a, b \in \mathbb{R}, a \neq 0 .
$$

If we choose $a=a_{0}^{-k}$ and $b=n b_{0} a^{-k}$ where $a_{0}>1, b_{0}>0$ and $n, k \in \mathbb{Z}^{+}$then we get the following family of discrete wavelets:

$$
\psi_{k, n}(t)=\left|a_{0}\right|^{-\frac{k}{2}} \psi\left(a_{0}^{k} t-n b_{0}\right) .
$$

These family of functions are a wavelet basis for $L^{2}(\mathbb{R})$ and makes an orthonormal basis for the special case $a_{0}=2$ and $b_{0}=1$.

Chebyshev wavelets $\psi_{n, m}(t)=\psi(k, m, n, t)$ have four arguments, $k=0,1,2, \cdots$, $n=1,2, \cdots, 2^{k}, m$ is the degree of Chebyshev polynomial of first kind and $t$ denotes the normalized time. They are defined on the interval $[0,1)$ by

$$
\psi_{n, m}(t)= \begin{cases}\frac{\alpha_{m} 2^{k / 2}}{\sqrt{\pi}} T_{m}\left(2^{k+1} t-2 n+1\right), & \frac{n-1}{2^{k}} \leq t \leq \frac{n}{2^{k}} \\ 0, & \text { otherwise }\end{cases}
$$

where

$$
\alpha_{m}= \begin{cases}\sqrt{2}, & m=0 \\ 2, & m=1,2, \cdots\end{cases}
$$

$T_{m}(t)$ in (4) are well known Chebyshev polynomial of order $m$, which is orthogonal with respect to the weight function $\omega(t)=\frac{1}{\sqrt{1-t^{2}}}$ and satisfy the following recursive formula:

$$
\begin{aligned}
& T_{0}(t)=1 \\
& T_{1}(t)=t
\end{aligned}
$$




$$
T_{m+1}(t)=2 t T_{m}(t)-T_{m-1}(t), \quad m=1,2,3, \cdots .
$$

Moreover, the set of Chebyshev wavelet are an orthogonal set with respect to the weight function $\omega_{n}(t)=\omega\left(2^{k+1} t-2 n+1\right)$.

Any function $f(t) \in L^{2}[0,1]$ may be expanded in terms of Chebyshev wavelet as

$$
f(t)=\sum_{n=1}^{\infty} \sum_{m=0}^{\infty} c_{n m} \psi_{n m}(t)
$$

where the wavelet coefficients of the series representation in (5) become

$$
c_{n m}=\left\langle f(t), \psi_{n m}(t)\right\rangle_{w_{n}(t)} .
$$

If the infinite series in (5) is truncated then Equation (5) can be written as

$$
f(t) \cong \sum_{n=1}^{2^{k-1}} \sum_{m=0}^{M-1} c_{n m} \psi_{n m}(t)=C^{\mathrm{T}} \Psi(t)
$$

where $C$ and $\Psi(t)$ are $2^{k-1} M \times 1$ matrices given by:

$$
\begin{aligned}
& C=\left[c_{1,0}, c_{1,1}, \cdots, c_{1, M-1}, c_{2,0}, c_{2,1}, \cdots, c_{2, M-1}, \cdots, c_{2^{k-1}, 0}, \cdots, c_{2^{k-1} M-1}\right]^{\mathrm{T}}, \\
& \Psi(t)=\left[\psi_{1,0}, \psi_{1,1}, \cdots, \psi_{1, M-1}, \psi_{2,0}, \psi_{2,1}, \cdots, \psi_{2, M-1}, \cdots, \psi_{2^{k-1}, 0}, \cdots, \psi_{2^{k-1}, M-1}\right]^{\mathrm{T}} .
\end{aligned}
$$

\section{Chebyshev Wavelets Operational Matrix of Derivative}

In this section, we first derive the operational matrix $D$ of derivative which plays a great role in order to reducing the given problem into solving the system of algebraic equation. For this, we concern with some Theorem and Corollary as follows.

Theorem 1 [24]. Let $\Psi(t)$ be the Chebyshev wavelets vector defined in (9), then we have

$$
\frac{\mathrm{d} \Psi(t)}{\mathrm{d} t}=D \Psi(t),
$$

where $D$ is $2^{k}(M+1)$ operational matrix of derivative as follows:

$$
D=\left[\begin{array}{cccc}
F & O & \ldots & O \\
O & F & \ldots & O \\
\vdots & \vdots & \ddots & \vdots \\
O & O & \ldots & F
\end{array}\right],
$$

in which $O$ is an $(M-1) \times(M+1)$ zero matrix, $F$ is an $(M+1)(M+1)$ matrix and its $(i, j)$ th element is defined as follows:

$$
F_{i, j}=\left\{\begin{array}{lc}
2^{k+1} \sqrt{(2 r-1)(2 s-1)}, & i=2, \cdots,(M+1), j=1, \cdots, i-1 \text { and }(i+j) \text { is odd } \\
0, & \text { otherwise. }
\end{array}\right.
$$

Corollary 1. By using Equation (10), the operational matrix for $n$th derivative can be derived as

$$
\frac{\mathrm{d}^{n} \Psi(t)}{\mathrm{d} t^{n}}=D^{n} \Psi(t)
$$


where $D^{n}$ is the $n t h$ power of matrix $D$.

\section{Chebyshev Wavelets Spectral Collocation Method}

In different type of numerical methods, spectral methods are one of the most popular methods of discretization for the numerical solution of partial differential equations and integral equations. The main advantage of this method lies in their accuracy for a given number of unknowns. For smooth problems in simple geometries, they offer exponential rates of convergence or spectral accuracy. In the recent literature, Galerkin, collocation, and Tau methods are the three most widely used spectral versions, in which collocation methods have become increasingly popular for solving differential equations, also they are very useful in providing highly accurate solutions to nonlinear differential equations. Now, we focus on the solution nature of this method as follows:

Let us consider the equation in the form:

$$
u_{t t}-\alpha u_{x x}+g(u)=f(x, t), \quad x \in\left[x_{L}, x_{R}\right], t \geq 0,
$$

with the initial conditions

$$
u(x, 0)=g_{1}(x), \frac{\partial}{\partial t} u(x, 0)=g_{2}(x), x \in \Omega, t \geq 0
$$

or boundary conditions

$$
u(x, t)=h(x, t), x \in \partial \Omega, t>0 .
$$

In order to transform the arbitrary domain $x_{L} \leq x \leq x_{R}$ into the domain defined for Chebyshev wavelet basis $0 \leq x \leq 1$, on can use the translation

$$
x:\left[x_{L}, x_{R}\right] \rightarrow[0,1], \quad X(x)=\frac{x-x_{L}}{x_{R}-x_{L}}
$$

By employing $\theta$-weight scheme [20], discreting the Equation (13), we can get

$$
\frac{u^{n+1}-2 u^{n}+u^{n-1}}{(\Delta t)^{2}}=\theta\left(\alpha \frac{\partial^{2} u^{n+1}}{\partial x^{2}}\right)-(1-\theta)\left(\alpha \frac{\partial^{2} u^{n}}{\partial x^{2}}\right)+g\left(u^{n}\right)=f^{n}, \quad 0<\theta \leq 1,
$$

where $\Delta t$ is the time step size with the expression $u^{n}(x)=u\left(t_{n}, x\right), t_{n}=n \times \Delta t$.

Now Equation (16) becomes

$$
\begin{aligned}
& u^{n+1}-\theta(\Delta t)^{2}\left(\alpha \frac{\partial^{2} u^{n+1}}{\partial x^{2}}\right) \\
& =2 u^{n}+(1-\theta)(\Delta t)^{2}\left(\frac{\partial^{2} u^{n}}{\partial x^{2}}\right)-(\Delta t)^{2} g\left(u^{n}\right)+(\Delta t)^{2} f^{n}-u^{n-1} .
\end{aligned}
$$

In the light of Equation (7), the term $u^{n}$ can be expanded by Chebyshev wavelet as

$$
u^{n}(x)=C_{n}^{\mathrm{T}} \Psi(x)
$$

Submitting Equation (18) into Equation (17), we have

$$
C_{n+1}^{\mathrm{T}} H_{L} \psi(x)=\left(C_{n}^{\mathrm{T}} H_{R}-C_{n-1}^{\mathrm{T}}\right) \psi(x)-(\Delta t)^{2} g\left(C_{n}^{\mathrm{T}} \psi(x)\right)+(\Delta t)^{2} f^{n},
$$

in which $H_{L}=I-\alpha \theta(\Delta t)^{2} D^{2}$ and $H_{R}=2 I+\alpha(1-\theta)(\Delta t)^{2} D^{2}$, where $D$ is the deri- 
vative matrix taken from Equation (10)

Also, by using the boundary conditions given in Equation (15), one can get

$$
C_{n+1}^{\mathrm{T}} \Psi(0)=h\left(0, t_{n+1}\right) \text { and } C_{n+1}^{\mathrm{T}} \Psi(1)=h\left(1, t_{n+1}\right) .
$$

Collocating Equation (19) in $2^{k-1} M-2$ Gauss-Chebyshev points $\left\{x_{i}\right\}_{i=2}^{2^{k-1} M-1}$, we have

$$
C_{n+1}^{\mathrm{T}} H_{L} \Psi\left(x_{i}\right)=\left(C_{n}^{\mathrm{T}} H_{R}-C_{n-1}^{\mathrm{T}}\right) \Psi\left(x_{i}\right)-(\Delta t)^{2} g\left(C_{n}^{\mathrm{T}} \Psi\left(x_{i}\right)\right)+(\Delta t)^{2} f^{n}\left(x_{i}, t_{n}\right) .
$$

Equation (20) and (21) can be written as matrix form

$$
A C_{n+1}=B \text {, }
$$

where $A$ and $B$ are $2^{k-1} M \times 2^{k-1} M$ and $2^{k-1} M \times 1$ matrices, respectively.

Again using the first and second initial conditions given in Equation (14), we have

$$
C_{0}^{\mathrm{T}} \Psi(x)=g_{i}(x), x \in \Omega
$$

and

$$
\frac{u^{1}-u^{-1}}{2 \Delta t}=g_{2}(t), x \in \Omega
$$

Equation (24) can be written as

$$
C_{-1}^{\mathrm{T}} \Psi(x)=C_{1}^{\mathrm{T}} \Psi(x)-2 \Delta \operatorname{tg}_{2}(x)
$$

Equation (22) using Equation (23) gives a linear system of equations with $2^{k-1} M$ unknown and equations, which can be solved to find $C_{n+1}$ in each step $n=0,1,2, \cdots$ so the unknown function $u\left(x, t_{n}\right)$ in any time $t=t_{n}$ can be found. Moreover, we defined the error bound for $\|e\|_{L_{2}}$ and $\|e\|_{L_{\infty}}$ as

$$
\|e\|_{L_{2}}=\sqrt{\sum_{j=1}^{M}\left|\left(e_{j}\right)^{2}\right|}, \quad\|e\|_{L_{\infty}}=\max _{j}\left|e_{j}\right|,
$$

where $e_{j}=\left(u_{\text {exact }}\right)_{j}-\left(u_{\text {approx }}\right)_{j}$ and $e=u_{\text {exact }}-u_{\text {approx }}$.

\section{Numerical Results and Discussions}

In this section, we use Chebyshev wavelets spectral collocation method described in section 4 to solve nonlinear type of Klein-Gordon and Sine-Gordon equations. The proposed method provides a reliable technique which is computer oriented if compared with traditional techniques. To give the clear overview of this method we consider three examples of Klein-Gordon equation and Sine-Gordon equation. All the results are calculated by using the symbolic calculus software MATLAB 2013a and Mathematica.

Example 1 [25] We consider the nonlinear Klein-Gordon Equation (13) with $\alpha=1$, $g(u)=u^{2}$ and $f(x, t)=-x \cos t+x^{2} \cos ^{2} t$ in the interval $[-1,1]$ with the initial conditions

$$
u(x, 0)=x, u_{t}(x, 0)=0, \quad-1 \leq x \leq 1
$$

and the Dirichlet boundary condition

$$
u(x, t)=h(x, t), \quad x \in \partial \Omega, t>0 .
$$


The analytical solution is given by

$$
f(x, t)=-x \cos t
$$

The obtained $L_{2}$ and $L_{\infty}$ errors of Example 1 at step size 0.0001 is presented in comparison with the existing method in Table 1 and Table 2 for $k=2, M=3$ and $k=2, M=4$ and graphically shown in Figure 1 for $k=2, M=4$. It is evident from Table 1, Table 2 and Figure 1 that the solutions obtain by using CWSCM are in good agreement and are better than the results obtained by existing method presented in [25]. However, the errors may be reduced significantly if we increase level of resolution.

Table 1. $L_{2}$ and $L_{\infty}$ error of Example 1 at $k=2, M=3$ and compared with [25].

\begin{tabular}{|c|c|c|c|c|c|c|}
\hline \multirow[b]{2}{*}{$t$} & \multicolumn{3}{|c|}{$L_{2}(M=3)$} & \multicolumn{3}{|c|}{$L_{\infty}(M=3)$} \\
\hline & CWSCM & MFDCM [25] & CM [25] & CWSCM & MFDCM [25] & CM [25] \\
\hline 0.1 & $3.1 \times 10^{-14}$ & $2.2 \times 10^{-12}$ & $1.7 \times 10^{-4}$ & $5.3 \times 10^{-12}$ & $3.5 \times 10^{-12}$ & $2.6 \times 10^{-4}$ \\
\hline 0.2 & $6.4 \times 10^{-14}$ & $8.2 \times 10^{-12}$ & $4.4 \times 10^{-4}$ & $4.8 \times 10^{-12}$ & $1.3 \times 10^{-11}$ & $3.5 \times 10^{-4}$ \\
\hline 0.3 & $4.0 \times 10^{-12}$ & $1.7 \times 10^{-11}$ & $3.6 \times 10^{-4}$ & $6.0 \times 10^{-12}$ & $2.5 \times 10^{-11}$ & $2.3 \times 10^{-4}$ \\
\hline 0.4 & $1.4 \times 10^{-13}$ & $2.7 \times 10^{-11}$ & $4.0 \times 10^{-4}$ & $4.1 \times 10^{-12}$ & $4.1 \times 10^{-11}$ & $3.1 \times 10^{-4}$ \\
\hline 0.5 & $9.6 \times 10^{-13}$ & $3.7 \times 10^{-11}$ & $4.2 \times 10^{-4}$ & $7.5 \times 10^{-12}$ & $5.7 \times 10^{-11}$ & $3.3 \times 10^{-4}$ \\
\hline 0.6 & $3.5 \times 10^{-13}$ & $4.7 \times 10^{-11}$ & $3.5 \times 10^{-4}$ & $8.9 \times 10^{-12}$ & $6.9 \times 10^{-11}$ & $2.6 \times 10^{-4}$ \\
\hline 0.7 & $2.9 \times 10^{-13}$ & $5.5 \times 10^{-11}$ & $3.8 \times 10^{-4}$ & $2.0 \times 10^{-12}$ & $6.8 \times 10^{-11}$ & $2.4 \times 10^{-4}$ \\
\hline 0.8 & $5.7 \times 10^{-13}$ & $6.0 \times 10^{-11}$ & $2.9 \times 10^{-4}$ & $5.4 \times 10^{-12}$ & $8.2 \times 10^{-11}$ & $3.0 \times 10^{-4}$ \\
\hline 0.9 & $3.7 \times 10^{-13}$ & $6.2 \times 10^{-11}$ & $2.7 \times 10^{-4}$ & $7.3 \times 10^{-12}$ & $8.1 \times 10^{-11}$ & $2.5 \times 10^{-4}$ \\
\hline 1.0 & $8.3 \times 10^{-12}$ & $5.9 \times 10^{-11}$ & $2.3 \times 10^{-4}$ & $5.2 \times 10^{-11}$ & $8.1 \times 10^{-11}$ & $2.2 \times 10^{-4}$ \\
\hline
\end{tabular}

Table 2. $L_{2}$ and $L_{\infty}$ error of Example 1 at $k=2, M=4$ and compared with [25].

\begin{tabular}{|c|c|c|c|c|c|c|}
\hline \multirow[b]{2}{*}{$t$} & \multicolumn{3}{|c|}{$L_{2}(M=4)$} & \multicolumn{3}{|c|}{$L_{\infty}(M=4)$} \\
\hline & CWSCM & MFDCM [25] & CM [25] & CWSCM & MFDCM [25] & CM [25] \\
\hline 0.1 & $6.4 \times 10^{-14}$ & $5.6 \times 10^{-14}$ & $4.1 \times 10^{-5}$ & $5.3 \times 10^{-13}$ & $6.7 \times 10^{-14}$ & $6.7 \times 10^{-5}$ \\
\hline 0.2 & $5.5 \times 10^{-14}$ & $9.6 \times 10^{-13}$ & $3.6 \times 10^{-5}$ & $1.3 \times 10^{-14}$ & $4.1 \times 10^{-12}$ & $4.2 \times 10^{-5}$ \\
\hline 0.3 & $5.4 \times 10^{-13}$ & $2.4 \times 10^{-12}$ & $4.9 \times 10^{-5}$ & $9.1 \times 10^{-13}$ & $3.8 \times 10^{-12}$ & $5.6 \times 10^{-5}$ \\
\hline 0.4 & $7.9 \times 10^{-13}$ & $4.7 \times 10^{-12}$ & $5.4 \times 10^{-5}$ & $7.5 \times 10^{-13}$ & $5.9 \times 10^{-12}$ & $7.3 \times 10^{-5}$ \\
\hline 0.5 & $8.1 \times 10^{-13}$ & $4.2 \times 10^{-12}$ & $6.3 \times 10^{-5}$ & $6.9 \times 10^{-13}$ & $5.8 \times 10^{-12}$ & $8.3 \times 10^{-5}$ \\
\hline 0.6 & $4.5 \times 10^{-13}$ & $4.8 \times 10^{-12}$ & $4.5 \times 10^{-5}$ & $1.4 \times 10^{-13}$ & $7.3 \times 10^{-12}$ & $6.6 \times 10^{-5}$ \\
\hline 0.7 & $4.7 \times 10^{-13}$ & $6.3 \times 10^{-12}$ & $5.8 \times 10^{-5}$ & $6.2 \times 10^{-13}$ & $8.2 \times 10^{-12}$ & $7.4 \times 10^{-5}$ \\
\hline 0.8 & $7.3 \times 10^{-13}$ & $7.1 \times 10^{-12}$ & $7.0 \times 10^{-5}$ & $8.9 \times 10^{-13}$ & $8.9 \times 10^{-12}$ & $8.1 \times 10^{-5}$ \\
\hline 0.9 & $9.4 \times 10^{-13}$ & $6.9 \times 10^{-12}$ & $5.2 \times 10^{-5}$ & $9.7 \times 10^{-13}$ & $8.8 \times 10^{-12}$ & $7.9 \times 10^{-5}$ \\
\hline 1.0 & $9.6 \times 10^{-13}$ & $6.4 \times 10^{-12}$ & $5.3 \times 10^{-5}$ & $4.1 \times 10^{-13}$ & $8.7 \times 10^{-12}$ & $7.7 \times 10^{-5}$ \\
\hline
\end{tabular}



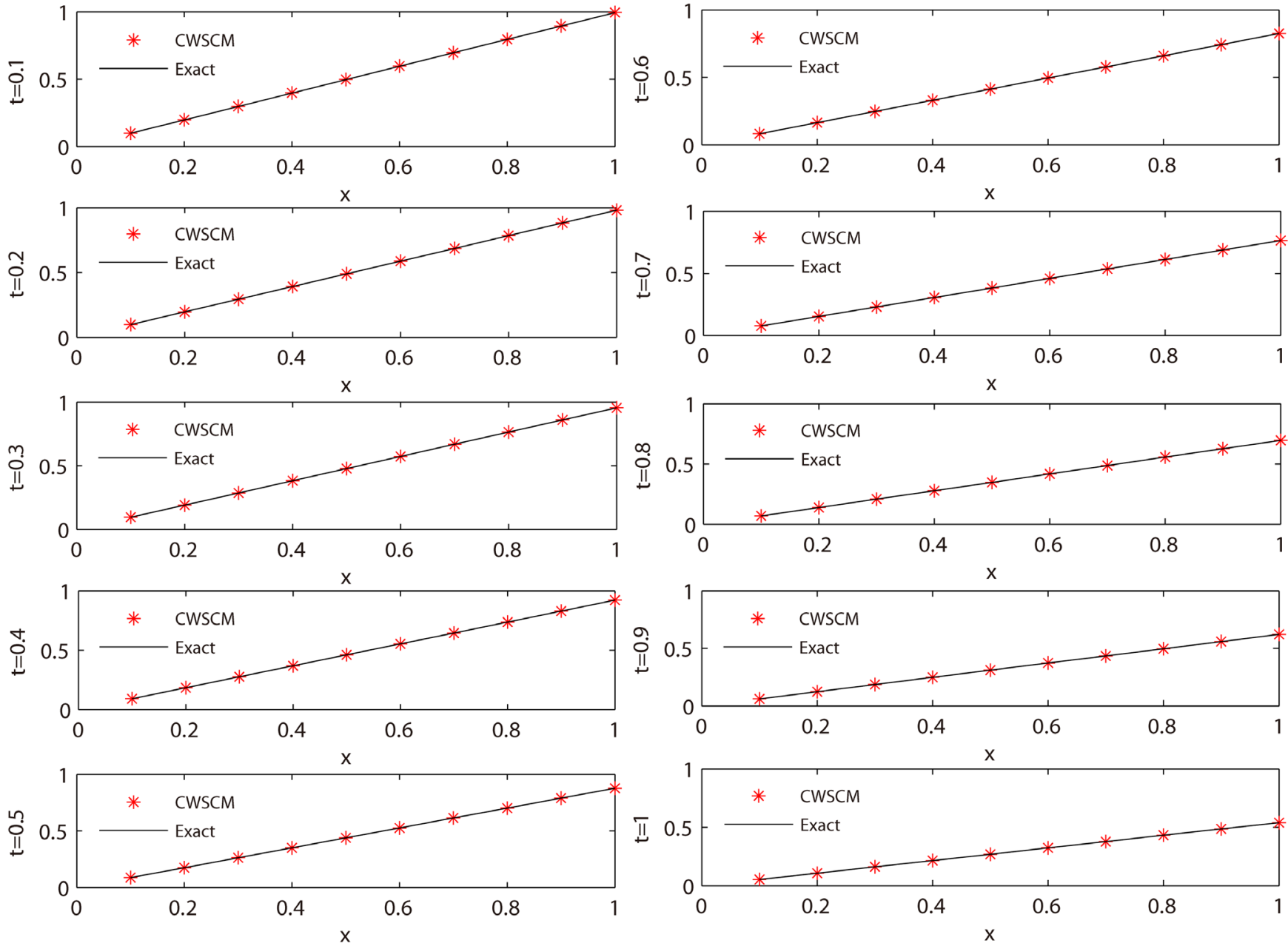

Figure 1. Comparison of exact solution with approximate solution for Example 1 at $k=2, M=4$.

Example 2 [25] We consider the nonlinear Klein-Gordon Equation (13) with $\alpha=1$, $g(u)=u^{2}$ and $f(x, t)=6 x t\left(x^{2}-t^{2}\right)+x^{6} t^{6}$ in the interval [0,1] with the initial conditions

$$
u(x, 0)=0, u_{t}(x, 0)=0,0 \leq x \leq 1
$$

and the Dirichlet boundary condition

$$
u(x, t)=h(x, t), x \in \partial \Omega, t>0 .
$$

The analytical solution is given by

$$
f(x, t)=x^{3} t^{3} .
$$

The $L_{2}$ and $L_{\infty}$ errors of Example 2 at step size 0.0001 are presented in comparison with the existing method in Table 3 and Table 4 for $k=2, M=3$ and $k=2$, $M=4$. From Table 3, Table 4 and Figure 2, it is clear that CWSCM performs much better than existing methods [25] and with the increase in number of collocation points the errors decrease for the solution. 
Table 3. $L_{2}$ and $L_{\infty}$ error of Example 2 at $k=2, M=3$ and compared with [25].

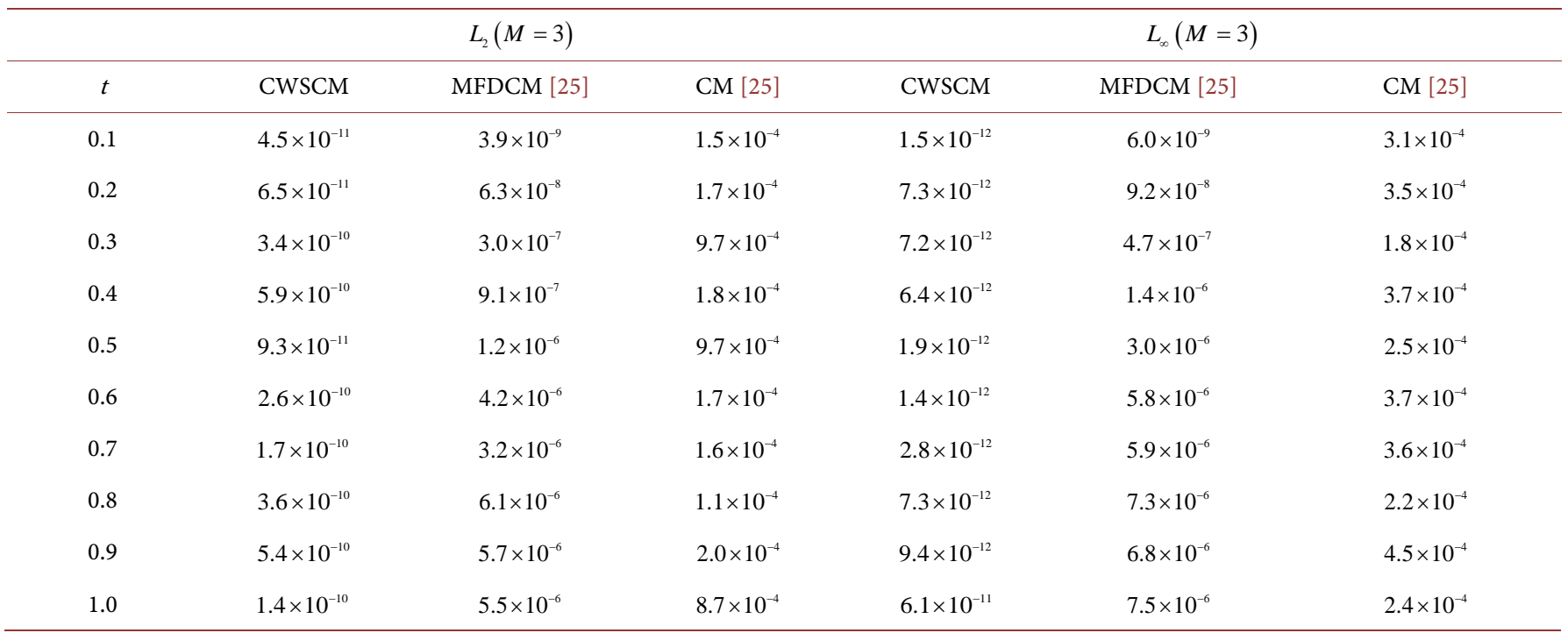

Table 4. $L_{2}$ and $L_{\infty}$ error of Example 2 at $k=2, M=4$ and compared with [25].

\begin{tabular}{|c|c|c|c|c|c|c|}
\hline$t$ & \multicolumn{3}{|c|}{$L_{2}(M=4)$} & \multicolumn{3}{|c|}{$L_{\infty}(M=4)$} \\
\hline 0.1 & $1.9 \times 10^{-11}$ & $4.4 \times 10^{-10}$ & $3.6 \times 10^{-5}$ & $3.2 \times 10^{-11}$ & $5.3 \times 10^{-10}$ & $5.3 \times 10^{-5}$ \\
\hline 0.2 & $4.3 \times 10^{-10}$ & $7.8 \times 10^{-9}$ & $3.9 \times 10^{-5}$ & $5.1 \times 10^{-11}$ & $9.4 \times 10^{-9}$ & $5.7 \times 10^{-5}$ \\
\hline 0.3 & $7.1 \times 10^{-9}$ & $4.5 \times 10^{-8}$ & $2.7 \times 10^{-5}$ & $9.0 \times 10^{-11}$ & $5.5 \times 10^{-8}$ & $4.1 \times 10^{-5}$ \\
\hline 0.5 & $6.0 \times 10^{-9}$ & $3.1 \times 10^{-7}$ & $3.2 \times 10^{-5}$ & $2.8 \times 10^{-9}$ & $5.6 \times 10^{-7}$ & $4.5 \times 10^{-5}$ \\
\hline 0.6 & $5.3 \times 10^{-9}$ & $5.6 \times 10^{-7}$ & $3.4 \times 10^{-5}$ & $7.9 \times 10^{-9}$ & $7.1 \times 10^{-7}$ & $5.9 \times 10^{-5}$ \\
\hline 0.7 & $9.1 \times 10^{-9}$ & $5.4 \times 10^{-7}$ & $3.5 \times 10^{-5}$ & $6.5 \times 10^{-9}$ & $7.0 \times 10^{-7}$ & $5.9 \times 10^{-5}$ \\
\hline 0.8 & $6.4 \times 10^{-9}$ & $6.8 \times 10^{-7}$ & $3.1 \times 10^{-5}$ & $5.2 \times 10^{-9}$ & $8.6 \times 10^{-7}$ & $4.5 \times 10^{-5}$ \\
\hline
\end{tabular}

Example 3 [20] Consider the following nonlinear Sine-Gordon equation

$$
\frac{\partial^{2} u}{\partial t^{2}}-\frac{\partial^{2} u}{\partial x^{2}}+\sin u=0,-10 \leq x \leq 10, t>0
$$

where $f(x, t)=0$, and the initial conditions

$$
u(x, 0)=0, u_{t}(x, 0)=4 \operatorname{sech}(x), 0 \leq x \leq 1
$$

and the Dirchlet boundary conditions

$$
u(x, t)=h(x, t), x \in \partial \Omega, t \geq 0 .
$$

The exact solution is given by

$$
u(x, t)=4 \tan ^{-1}(\operatorname{sech}(x) t)
$$



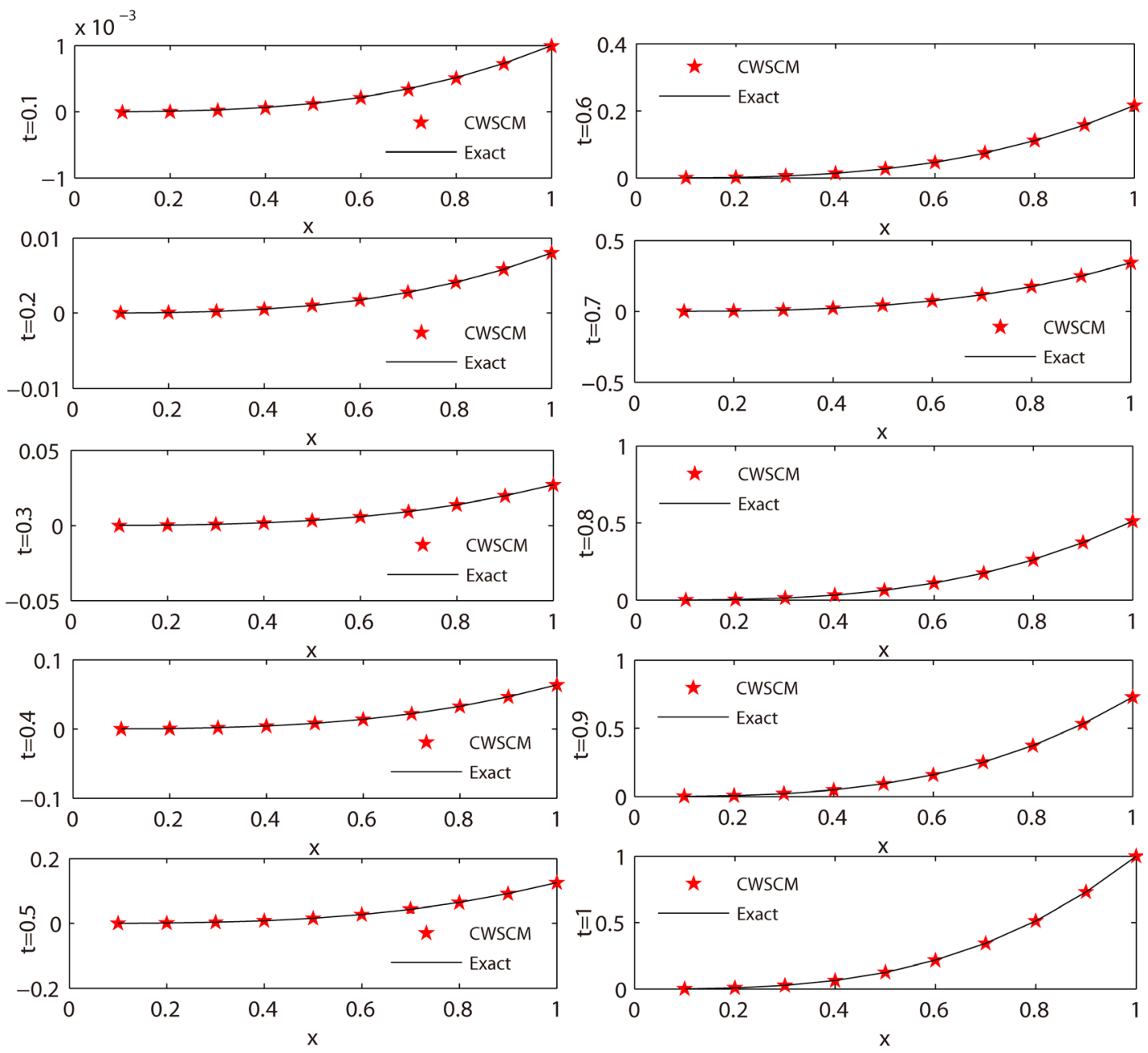

Figure 2. Comparison of exact solution with approximate solution for Example 2 at $k=2, M=4$.

The numerical solution of Sine-Gordon equation has presented in Table 5 which shows the comparison of the errors of the present method with the exact solution. It is obvious from the table that the present method is more accurate, simple and fast. Comparison between an exact and approximate solution is shown in Figure 3.

\section{Concluding Remarks}

In this article, we have proposed an efficient and accurate method based on Chebyshev wavelets to solve both Klein-Gordon and Sine-Gordon equations arising in different field of sciences, engineering and technology. The main advantage of this method is that it transforms the problem into algebraic equation so that the computation is effective and simple. To appraise the performance and efficiency of the method, three benchmark problems are included and discussed. The numerical results are compared with a few existing methods reported recently in the literature. The numerical experiments confirm that the spectral method coupled with Chebyshev wavelets is superior to other existing ones. 
Table 5. $L_{2}$ and $L_{\infty}$ error of Example 3 at $k=2, M=3$ and 4 .

\begin{tabular}{ccccc}
\hline & \multicolumn{2}{c}{ CWSCM $(k=2, M=3)$} & \multicolumn{2}{c}{ CWSCM $(k=2, M=4)$} \\
\hline$t$ & $L_{2}$ & $L_{\infty}$ & $L_{2}$ & $L_{\infty}$ \\
\hline 0.1 & $2.4 \times 10^{-10}$ & $8.3 \times 10^{-11}$ & $6.2 \times 10^{-10}$ & $7.4 \times 10^{-10}$ \\
0.2 & $5.7 \times 10^{-10}$ & $6.7 \times 10^{-11}$ & $4.1 \times 10^{-11}$ & $1.9 \times 10^{-9}$ \\
0.3 & $1.6 \times 10^{-9}$ & $2.9 \times 10^{-10}$ & $9.1 \times 10^{-10}$ & $4.3 \times 10^{-9}$ \\
0.4 & $8.0 \times 10^{-10}$ & $7.1 \times 10^{-9}$ & $1.7 \times 10^{-9}$ & $2.9 \times 10^{-9}$ \\
0.5 & $7.6 \times 10^{-9}$ & $6.1 \times 10^{-9}$ & $8.8 \times 10^{-8}$ & $9.6 \times 10^{-9}$ \\
0.6 & $4.3 \times 10^{-9}$ & $5.7 \times 10^{-9}$ & $7.1 \times 10^{-8}$ & $4.1 \times 10^{-9}$ \\
0.7 & $9.5 \times 10^{-9}$ & $4.4 \times 10^{-9}$ & $7.5 \times 10^{-8}$ & $7.4 \times 10^{-9}$ \\
0.8 & $9.4 \times 10^{-9}$ & $1.9 \times 10^{-9}$ & $5.8 \times 10^{-8}$ & $1.6 \times 10^{-9}$ \\
0.9 & $7.4 \times 10^{-9}$ & $4.2 \times 10^{-9}$ & $5.3 \times 10^{-8}$ & $7.5 \times 10^{-9}$ \\
1.0 & $9.6 \times 10^{-9}$ & $6.5 \times 10^{-9}$ & $2.2 \times 10^{-8}$ & $7.5 \times 10^{-8}$ \\
\hline
\end{tabular}
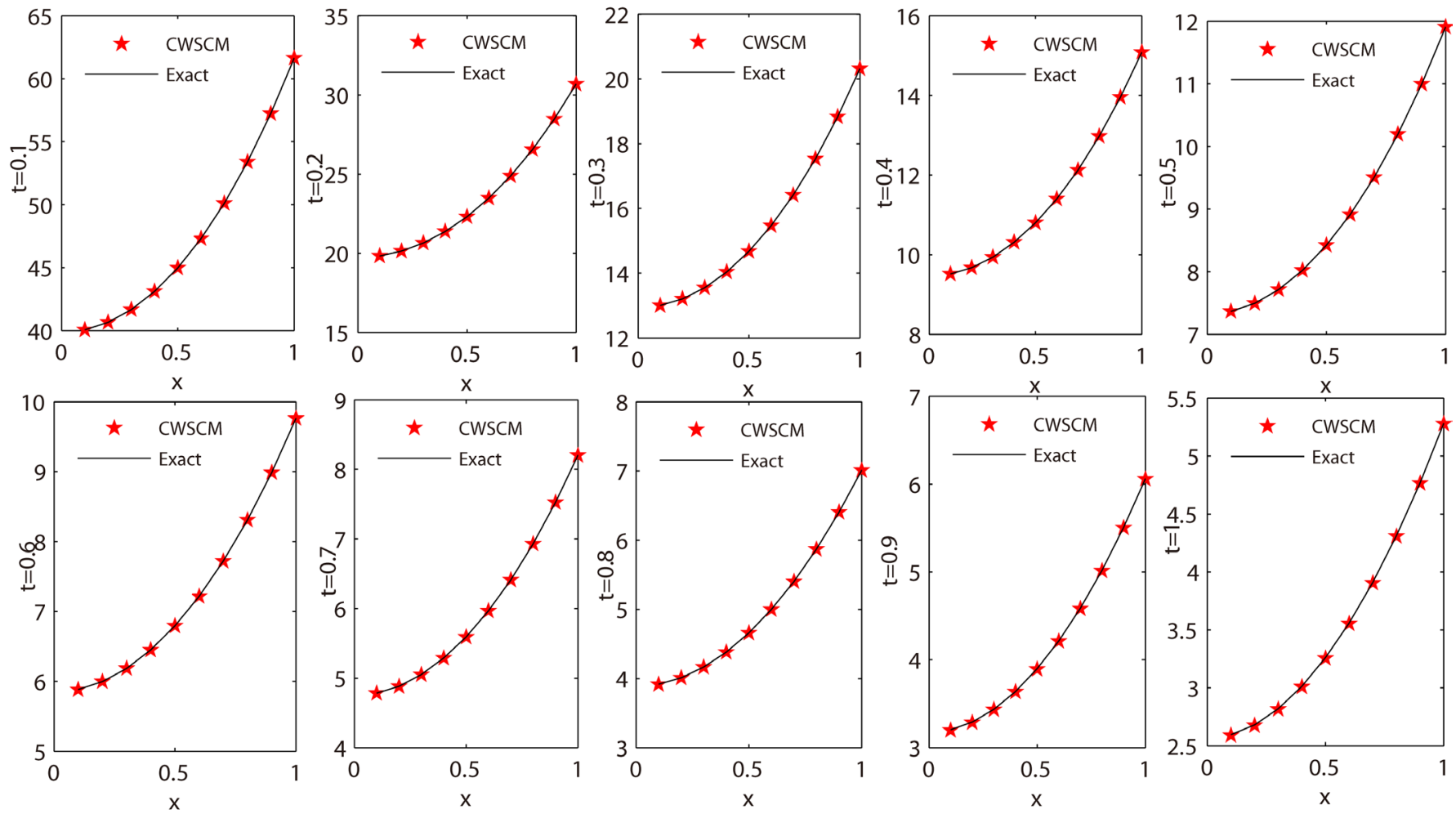

Figure 3. Comparison of exact solution with approximate solution for Example 3 at $k=2, M=4$.

\section{Acknowledgements}

We thank the Editor and the referee for their comments.

\section{References}

[1] Dehghan, M. and Shokri, A. (2009) Numerical Solution of the Nonlinear Klein-Gordon 
Equation Using Radial Basis Functions. Journal of Computational and Applied Mathematics, 230, 400-410. http://dx.doi.org/10.1016/j.cam.2008.12.011

[2] Dehghan, M., Mohebbi, A. and Asghari, Z. (2009) Fourth-Order Compact Solution of the Nonlinear Kleingordon Equation. Numerical Algorithms, 52, 523-540.

http://dx.doi.org/10.1007/s11075-009-9296-x

[3] Wazwaz, A.M. (2005) The Tanh and the Sine-Cosine Methods for Compact and Noncompact Solutions of the Nonlinear Klein-Gordon Equation. Applied Mathematics and Computation, 167, 1179-1195. http://dx.doi.org/10.1016/j.amc.2004.08.006

[4] Duncan, D.B. (1997) Symplectic Finite Difference Approximations of the Nonlinear KleinGordon Equation. SIAM Journal on Numerical Analysis, 34, 1742-1760.

http://dx.doi.org/10.1137/S0036142993243106

[5] Grella, G. and Marinaro, M. (1978) Special Solutions of the Sine-Gordon Equation in $2+1$ Dimensions. Lettere al Nuovo Cimento, 23, 459-464. http://dx.doi.org/10.1007/BF02770537

[6] Koshelev, A.E. (2010) Stability of Dynamic Coherent States in Intrinsic Josephson-Junction Stacks near Internal Cavity Resonance. Physical Review B, 82, 174-512. http://dx.doi.org/10.1103/PhysRevB.82.174512

[7] Krasnov, V.M. (2010) The Emission form Intrinsic Josephson Junctions at Zeros Magnetic Field via Breather Auto-Oscillations. Physical Review B, 83, 174-512.

[8] El-Sayed, S.M. (2003) The Decomposition Method for Studying the Klein-Gordon Equation. Chaos Solitons \& Fractals, 18, 1025-1030. http://dx.doi.org/10.1016/S0960-0779(02)00647-1

[9] Batiha, B., Noorani, M.S.M. and Hashim, I. (2007) Numerical Solution of Sine-Gordon Equation by Variational Iteration Method. Physics Letters A, 370, 437-440.

http://dx.doi.org/10.1016/j.physleta.2007.05.087

[10] Shakeri, F. and Dehghan, M. (2008) Numerical Solution of the Klein Gordon Equation via He's Variational Iteration Method. Nonlinear Dynamics, 51, 89-97. http://dx.doi.org/10.1007/s11071-006-9194-x

[11] Dehghan, M. and Shokri, A. (2008) A Numerical Method for One-Dimensional Nonlinear Sine-Gordon Equation Using Collocation and Radial Basis Functions. Numerical Methods for Partial Differential Equations, 24, 687-698. http://dx.doi.org/10.1002/num.20289

[12] Sirendaoreji (2007) Auxiliary Equation Method and New Solutions of Klein-Gordon Equations. Chaos, Solitons \& Fractals, 31, 943-950. http://dx.doi.org/10.1016/j.chaos.2005.10.048

[13] Guo, B.-Y., Xun, L. and Vazquez, L. (1996) A Legendre Spectral Method for Solving the Nonlinear Klein-Gordon Equation. The Journal of Computational and Applied Mathematics, 15, 19-36.

[14] Li, X. and Guo, B. Y. (1997) A Legendre Spectral Method for Solving the Nonlinear KleinGordon Equation. Journal of Computational Mathematics, 15, 105-126.

[15] Sweilam, N.H., et al. (2016) New Spectral Second Kind Chebyshev Wavelets Scheme for Solving Systems of Integro-Differential Equations. International Journal of Applied and Computational Mathematics, 4, 29-51.

[16] Hariharan, G. (2011) Haar Wavelet Method for Solving the Klein-Gordon and the SineGordon Equations. International Journal of Nonlinear Sciences, 11, 180-189.

[17] Yin, F., Song, J. and Lu, F. (2014) A Coupled Method of Laplace Transform and Legendre Wavelets for Nonlinear Klein-Gordon Equations. Mathematical Methods in the Applied Sciences, 37, 781-791. http://dx.doi.org/10.1002/mma.2834

[18] Iqbal J. and Abass R. (2016) Numerical Solution of Hammerstein Integral Equation Using 
Chebyshev Wavelet Method. Journal of Mathematical and Computational Science.

[19] Guo, B.Y., Li, X. and Vazquez, L. (1996) A Legendre Spectral Method for Solving the Nonlinear Klein-Gordon Equation. Applied Mathematics and Computation, 15, 19-36.

[20] Fukang, Y., et al. (2015) Spectral Methods Using Legendre Wavelets for Nonlinear Klein/ Sine-Gordon Equations. Journal of Computational and Applied Mathematics, 275, 321-334. http://dx.doi.org/10.1016/j.cam.2014.07.014

[21] Babolian, E. and Fattahzadeh, F. (2007) Numerical Solution of Differential Equations by Using Chebyshev Wavelet Operational Matrix of Integration. Applied Mathematics and Computation, 188, 417-426. http://dx.doi.org/10.1016/j.amc.2006.10.008

[22] Boggess, A. and Narcowich, F.J. (2001) A First Course in Wavelets with Fourier Analysis. John Wiley and Sons, Hoboken.

[23] Gu, J.S. and Jiang, W.S. (1996) The Haar Wavelets Operational Matrix of Integration. International Journal of Systems Science, 27, 623-628. http://dx.doi.org/10.1080/00207729608929258

[24] Hosseini, S.Gh. and Mohammadi, F. (2011) A New Operational Matrix of Derivative for Chebyshev Wavelets and Its Applications in Solving Ordinary Differential Equations with Non Analytic Solution. Applied Mathematical Sciences, 5, 2537-2548.

[25] Lakestani, M. and Dehghan, M. (2010) Collocation and Finite Difference-Collocation Methods for the Solution of Nonlinear Klein-Gordon Equation. Computer Physics Communications, 181, 1392-1401. http://dx.doi.org/10.1016/j.cpc.2010.04.006

\section{Submit or recommend next manuscript to SCIRP and we will provide best service} for you:

Accepting pre-submission inquiries through Email, Facebook, LinkedIn, Twitter, etc. A wide selection of journals (inclusive of 9 subjects, more than 200 journals)

Providing 24-hour high-quality service

User-friendly online submission system

Fair and swift peer-review system

Efficient typesetting and proofreading procedure

Display of the result of downloads and visits, as well as the number of cited articles

Maximum dissemination of your research work

Submit your manuscript at: http://papersubmission.scirp.org/

Or contact am@scirp.org 